\title{
APSA Organized Sections
}

\begin{tabular}{|c|c|c|c|c|}
\hline Name of Section & Contact or Officers & $\begin{array}{l}\text { Annual Meeting } \\
\text { Program Organizer }\end{array}$ & Dues & Estab. \\
\hline $\begin{array}{l}\text { 1. Federalism and } \\
\text { Intergovernmental } \\
\text { Relations }\end{array}$ & $\begin{array}{l}\text { Chair: Ellis Katz, Dept. of } \\
\text { Pol. Sci., Temple Univ., } \\
\text { Philadelphia, PA 19122; } \\
\text { (215) 787-I482 } \\
\text { Secretary-Treasurer: E. } \\
\text { Lester Levine, Empire } \\
\text { State College, SUNY, } \\
564 \text { Franklin St., Buffalo, } \\
\text { NY I4202; (716) } \\
886-8020 \\
\text { Newsletter Editor: Ellis } \\
\text { Katz } \\
\text { Executive Council; John } \\
\text { Kincaid, U.S. ACIR; Ellis } \\
\text { Katz, Temple Univ.; } \\
\text { Beverly Cigler, Penn } \\
\text { State at Harrisburg; } \\
\text { Margaret Wrightson, } \\
\text { Falls Church. VA; Dale } \\
\text { Krane, Univ. of } \\
\text { Nebraska; Donald Lutz, } \\
\text { Univ. of Houston; Daniel } \\
\text { J. Elazar, Temple Univ.; } \\
\text { Kenneth Palmer, Univ. of } \\
\text { Maine; Robert Dilger. } \\
\text { Univ. of Redlands }\end{array}$ & $\begin{array}{l}\text { Robert Thomas, Dept. of } \\
\text { Pol. Sci., Univ. of } \\
\text { Houston, Houston, TX } \\
77204-3474 \text {; (7 I 3) 749- } \\
4887 \text { and Ellis Katz }\end{array}$ & $\$ 5.00$ & $9 / 83$ \\
\hline $\begin{array}{l}\text { 2. Law, Courts, and } \\
\text { Judicial Process }\end{array}$ & $\begin{array}{l}\text { Chair: Karen O'Connor, } \\
\text { Emory Univ., Pol. Sci., } \\
\text { Atlanta, GA } 30309 ; \\
\text { (404) 727-7910 } \\
\text { Secretary-Treasurer: } \\
\text { Victor Flango, National } \\
\text { Center for State Courts, } \\
300 \text { Newport Ave., } \\
\text { Williamsburg, VA } 23187 . \\
\text { 8798; (804) 253-2000 } \\
\text { Newsletter Editor: } \\
\text { William P. McLauchlan, } \\
\text { Purdue Univ., Dept. } \\
\text { of Pol. Sci., W. } \\
\text { Lafayette, IN 47907; } \\
\text { (317) 494-4171 } \\
\text { President-Elect: Stuart A. } \\
\text { Scheingold, Univ. of } \\
\text { Washington, Dept. of } \\
\text { Pol. Sci., Gowan Hall, } \\
\text { DO-30, Seattle, WA } \\
\text { 98195; (206) 543-2377 }\end{array}$ & Karen O'Conner & $\$ 5.00$ & $9 / 83$ \\
\hline
\end{tabular}




\section{Gazette}

\begin{tabular}{|c|c|c|c|c|}
\hline Name of Section & Contact or Officers & $\begin{array}{l}\text { Annual Meeting } \\
\text { Program Organizer }\end{array}$ & Dues & Estab. \\
\hline 3. Legislative Studies & 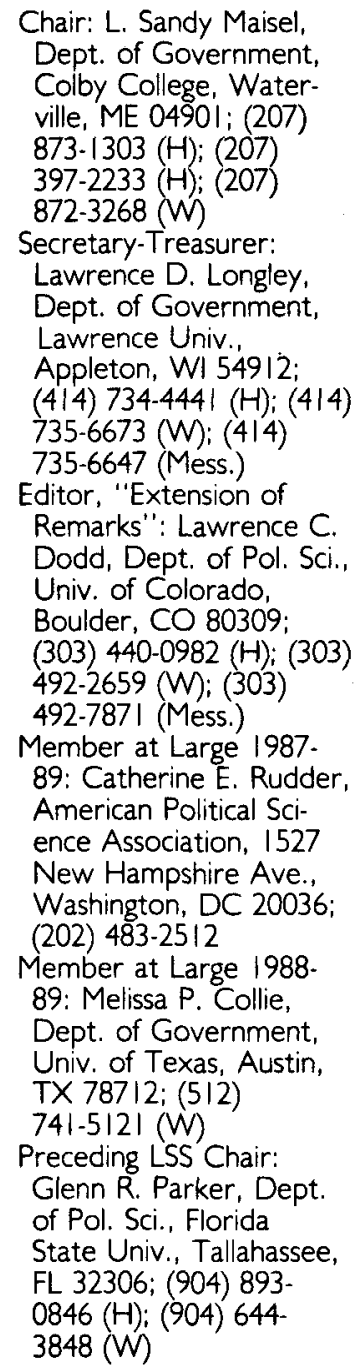 & $\begin{array}{l}\text { Alan Abramovitz, Dept. } \\
\text { of Pol. Sci., Emory Univ., } \\
\text { Atlanta, GA 30322; } \\
\text { (404) 727-0108 (W) }\end{array}$ & $\$ 5.00$ & $9 / 83$ \\
\hline
\end{tabular}

4. Policy Studies President: Rita Mae Kelly, School of Justice Studies,

Arizona State Univ.,

Tempe, AZ 84287; (602)

$831-2283(H) ;(602)$

965-4529 (W)

President-Elect and Vice

President: Daniel Maz-

manian, Center for Pol.

and Policy, Claremont

Grad. School, 170 E.

10th St., Claremont, CA

971।1-6163; (714) 621.

$8284(W)$ 


\begin{tabular}{|c|c|c|c|c|}
\hline Name of Section & Contact or Officers & $\begin{array}{l}\text { Annual Meeting } \\
\text { Program Organizer }\end{array}$ & Dues & Estab. \\
\hline & $\begin{array}{l}\text { Secretary-Treasurer: } \\
\text { Stuart S. Nagel, Univ. of } \\
\text { Illinois. Dept. of Pol. Sci., } \\
702 \text { S. Wright St., } \\
\text { Urbana, IL 61801; (217) } \\
\text { 333-3880 }\end{array}$ & & & \\
\hline $\begin{array}{l}\text { 5. Political Parties } \\
\text { and Organizations }\end{array}$ & $\begin{array}{l}\text { Chair: Frank J. Sorauf, } \\
\text { Dept. of Pol. Sci.. Univ. } \\
\text { of Minnesota, I414 } \\
\text { Social Sciences Bldg., } \\
\text { Minneapolis, MN } 55455 ; \\
\text { (612) } 624-5503 \\
\text { Newsletter Editor: } \\
\text { (position vacant) }\end{array}$ & $\begin{array}{l}\text { James L. Gibson, Pol. Sci. } \\
\text { Dept., Univ. of Houston, } \\
\text { Houston, TX } 77004 ; \\
\text { (7I3) } 660-8813(\mathrm{H}) \\
\text { (7I3) } 749-4322(\mathrm{~W})\end{array}$ & $\$ 5.00$ & $9 / 83$ \\
\hline $\begin{array}{l}\text { 6. Public } \\
\text { Administration }\end{array}$ & $\begin{array}{l}\text { Chair: Barbara Romzek, } \\
\text { Dept. of Public Admin., } \\
\text { Univ. of Kansas, Blake } \\
\text { Hall, Lawrence, KS } \\
\text { 66045; (9|3) 864-3527 } \\
\text { Treasurer: Robert Mount- } \\
\text { joy, Dept. of Pol. Sci., } \\
\text { Auburn Univ., Auburn, } \\
\text { AL 36|93; (205) 826- } \\
5370 \text { (W) } \\
\text { Newsletter Editor: } \\
\text { William L. Waugh, Inst. } \\
\text { of Pub. Admin., Georgia } \\
\text { State Univ., Atlanta, GA } \\
\text { 30303; (404) 658-3350 } \\
\text { (W) }\end{array}$ & $\begin{array}{l}\text { Donald F. Kettl, Dept. of } \\
\text { Gov. and Foreign Affairs, } \\
\text { Univ. of Virginia, Char- } \\
\text { lottesville, VA 22903; } \\
\text { (804) 924-3358 (W) }\end{array}$ & & \\
\hline 7. Conflict Processes & $\begin{array}{l}\text { Chair: Jacek Kugler, Pol. } \\
\text { Sci. Dept., Vanderbilt } \\
\text { Univ., Nashville, TN } \\
37235 ;(6 \mid 5) 322-6239 \\
\text { (W) } \\
\text { Executive Committee: } \\
\text { Manus Midlarsky, Univ. } \\
\text { of Florida, Tallahassee; } \\
\text { Bruce Bueno de } \\
\text { Mesquita, Stanford } \\
\text { Univ.: William Thomp- } \\
\text { son. Claremont Univ.; } \\
\text { Randall Siverson, Univ. } \\
\text { of Calif., Davis; Bingham } \\
\text { Powell, Univ. of Roches- } \\
\text { ter; Frank Zagare, } \\
\text { SUNY. Buffalo; Robert } \\
\text { Jackman, Michigan State } \\
\text { Univ. }\end{array}$ & Jacek Kugler & $\$ 5.00$ & $8 / 84$ \\
\hline
\end{tabular}




\begin{tabular}{|c|c|c|c|c|}
\hline Name of Section & Contact or Officers & $\begin{array}{l}\text { Annual Meeting } \\
\text { Program Organizer }\end{array}$ & Dues & Estab. \\
\hline $\begin{array}{l}\text { 8. Representation and } \\
\text { Electoral Systems }\end{array}$ & $\begin{array}{l}\text { Chair: Joseph Zimmer- } \\
\text { man, Dept. of Pol. Sci., } \\
\text { State Univ. of New } \\
\text { York. Albany. Albany, } \\
\text { NY } 12222 ;(5 / 8) 439 . \\
9440(\mathrm{H}) \text {; (5 I8) 442-- } \\
5378(\mathrm{~W}) \\
\text { Executive Committee: } \\
\text { Bernard Grofman, Univ. } \\
\text { of Calif., Irvine; } \\
\text { Lawrence Longley, } \\
\text { Lawrence Univ.; Theo- } \\
\text { dore Robinson, Louisiana } \\
\text { State Univ.; Wilma Rule, } \\
922 \text { Glidden Ave., De } \\
\text { Kalb, IL; Joseph Zim- } \\
\text { merman } \\
\text { Newsletter Editor: Joseph } \\
\text { Zimmerman }\end{array}$ & Joseph Zimmerman & $\$ 5.00$ & $8 / 84$ \\
\hline
\end{tabular}

9. Presidency Research Chair: Erwin C. Hargrove, Terry Moe, Stanford

Jr., Vanderbilt Univ., Political Science, Box 1714. Sta. B, Nashville, TN 37205; (615)

322.6234

Secretary-Treasurer: Michael Grossman, clo Martha Kumar, Dept. of Pol. Sci., Towson State Univ., Baltimore, MD 21204: (30I) 321-2958 Vice President and President-Elect: Betty Glad, Univ. of lilinois (Univ. of So. Carolina, Columbia) Neustadt Award Comm. Chair: Samuel Kernell. Pol. Sci., Univ. of Calif., San Diego

Newsletter Editor: Cary

Covington, Univ. of lowa; Pol. Sci., lowa City, IA 52242; (319) 335-2339

\begin{tabular}{|c|c|c|c|}
\hline $\begin{array}{l}\text { 10. Political } \\
\text { Methodology }\end{array}$ & $\begin{array}{l}\text { Chair: Stanley Feldman, } \\
\text { Dept. of Pol. Sci., Univ. } \\
\text { of Kentucky, Lexington, } \\
\text { KY 40506; (606) 257. } \\
7050 \\
\text { Secretary-Treasurer: } \\
\text { Nathaniel Beck, Dept. of } \\
\text { Pol. Sci., Univ. of Calif., } \\
\text { San Diego, La Jolla, } \\
\text { CA 92093; (714) } \\
452-3548\end{array}$ & $\begin{array}{l}\text { Henry Brady, Univ. of } \\
\text { Chicago, Chicago, IL } \\
60637 ;(312) 702-0900 ; \\
(312) 667-7441 \text { (W) }\end{array}$ & $\$ 5.00$ \\
\hline
\end{tabular}
Univ., Dept. of Pol 94305; (415) 723-1806 (W) Sci. Stanford, CA 


\begin{tabular}{ll} 
Name of Section & Contact or Officers \\
\hline & Newsletter Editor: Gary \\
King, Dept. of Govern- & ment, Harvard Univ. \\
Cambridge, MA 02138; \\
(617) 495-2148
\end{tabular}

11. Religion and Politics Chair: Clarke E. Cochran Dept. of Pol. Sci., Texas Tech. Univ., Lubbock, TX 79409-4290; (806) 795-9745

Vice Chair: Lyman Kellstedt, Dept. of Pol. Sci., Wheaton College, Wheaton, IL 60187 ; (312) 260-5129

Treasurer: Clarke E. Cochran

Newsletter Editor: Kenneth Wald, Dept. of Pol. Sci., Univ. of Florida, Gainesville, FL 3261 I; (904) 392-0262

Steering Committee: Mary Seegers, Rutgers; Stephen V. Monsma, Pepperdine Univ.; Jo Renee Formicola, Seton Hall Univ.; Daniel J. O'Neil, Univ. of Arizona

12. Politics and Life Sciences

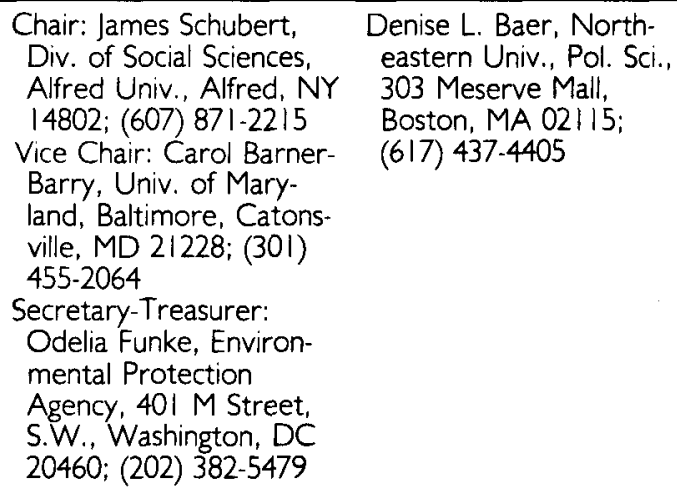

Chair: James Schubert, Div. of Social Sciences, Alfred Univ., Alfred, NY 14802; (607) 871-2215

Vice Chair: Carol BarnerBarry, Univ. of Maryland, Baltimore, Catonsville, MD 21228; (301) 455-2064

Secretary-Treasurer: Odelia Funke, Environmental Protection Agency, 401 M Street, S.W., Washington, DC 20460; (202) 382-5479

Denise L. Baer, Northeastern Univ., Pol. Sci., 303 Meserve Mall. Boston, MA 02115; (617) 437.4405

Clarke E. Cochran

(1990): Lyman Kellstedt

Chair: Clarence N. Stone, Clarence N. Stone Univ. of Maryland, Gov. and Pol., College Park, MD 20742; (301) 4546720

Secretary-Treasurer: John Pelissero, Dept. of Pol. Sci., Loyola Univ. of Chicago; (312) 508-3066 


\begin{tabular}{|c|c|c|c|c|}
\hline Name of Section & Contact or Officers & $\begin{array}{l}\text { Annual Meeting } \\
\text { Program Organizer }\end{array}$ & Dues & Estab. \\
\hline & $\begin{array}{l}\text { (W); (312) 670-31 I0 } \\
\text { (Mess.) } \\
\text { Newsletter Editor: Ken } \\
\text { Mladenka, Pol. Sci. } \\
\text { Dept., Texas A \& M, } \\
\text { College Station, TX } \\
\text { 77843; (409) 845-25II } \\
\text { Executive Committee: } \\
\text { Pres.: Brian D. Jones, } \\
\text { Texas A \& M; Elinor } \\
\text { Ostrom, Indiana Univ.; } \\
\text { Dianne Pinderhughes, } \\
\text { Univ. of Illinois; Susan } \\
\text { Welch, Univ. of } \\
\text { Nebraska; Mark } \\
\text { Schneider, SUNY. Stony } \\
\text { Brook; Susan Clarke, } \\
\text { Univ. of Colorado; } \\
\text { Steven Erie, Univ. of } \\
\text { Calif., San Diego; William } \\
\text { Nelson, Ohio State } \\
\text { Univ.; Harold Wolman, } \\
\text { Wayne State Univ.; Ann } \\
\text { Bowman, Univ. of So. } \\
\text { Carolina; Alberta } \\
\text { Sbragia, Univ. of } \\
\text { Pittsburgh }\end{array}$ & & & \\
\hline $\begin{array}{l}\text { 14. Applied Political } \\
\text { Science }\end{array}$ & $\begin{array}{l}\text { Chair: Howard Silver, } \\
\text { Consortium for Social } \\
\text { Science Associations, } \\
1625 \text { I St., N.W., Wash- } \\
\text { ington, DC 20006; (202) } \\
887-6166 \\
\text { Secretary-Treasurer: Her- } \\
\text { bert Smith, Dept. of Pol. } \\
\text { Sci., W. Maryland Coll., } \\
\text { Westminster, MD } 21157 \text {; } \\
\text { (301) 848-7000 × } 413\end{array}$ & Howard Silver & $\$ 5.00$ & $4 / 86$ \\
\hline $\begin{array}{l}\text { 15. Science and } \\
\text { Technology Studies }\end{array}$ & $\begin{array}{l}\text { Chair: Edward Wood- } \\
\text { house, Dept. of Sci. and } \\
\text { Tech. Studies, Rensselaer } \\
\text { Polytech. Inst., Troy, NY } \\
12180-3590 ;(518) \\
276-8506 \\
\text { Secretary-Treasurer: } \\
\text { Patrick Hamlett, Dept. } \\
\text { of History and Pol. Sci., } \\
\text { Univ. of Missouri, Rolla, } \\
\text { Rolla, MO 6540I; (314) } \\
341-4805 \\
\text { Newsletter Editor: Jon } \\
\text { Alexander, American } \\
\text { Gov. and Pol., Carleton } \\
\text { Univ., Ottawa, Ontario } \\
\text { KIS 5B6, Canada; (6I3) } \\
564-2697\end{array}$ & $\begin{array}{l}\text { Norman J. Vig, Dept. of } \\
\text { Pol. Sci., Carleton Col- } \\
\text { lege, Northfield, MN } \\
\text { 55057; (507) 663-4120 }\end{array}$ & $\$ 8.00$ & $8 / 86$ \\
\hline
\end{tabular}




\begin{tabular}{|c|c|c|c|c|}
\hline Name of Section & Contact or Officers & $\begin{array}{l}\text { Annual Meeting } \\
\text { Program Organizer }\end{array}$ & Dues & Estab. \\
\hline 16. Women and Politics & $\begin{array}{l}\text { President: Susan Welch, } \\
\text { Dept. of Pol. Sci., Univ. } \\
\text { of Nebraska, Lincoln, NE } \\
68588 ;(402) 472-5704 \\
\text { President-Elect: Jane } \\
\text { Mansbrioge, Dept. of } \\
\text { Pol. Sci., Northwestern } \\
\text { Univ., 2040 Sheridan } \\
\text { Rd., Evanston, IL 60201; } \\
\text { (312) 49I-8726 } \\
\text { Secretary-Treasurer: } \\
\text { Anne Costain, Dept. of } \\
\text { Pol. Sci., Univ. of Colo- } \\
\text { rado, Boulder, Campus } \\
\text { Box 333, Boulder, CO } \\
\text { 80309; (303) 492-787I } \\
\text { Executive Council: } \\
\text { Pamela Conover, Univ. } \\
\text { of No. Carolina; Bob } \\
\text { Darcy, Oklahoma State } \\
\text { Univ.; Dorothy Stetson, } \\
\text { Florida Atlantic Univ.; } \\
\text { Pinky Wassenberg, } \\
\text { Sangamon State Univ. }\end{array}$ & Susan Welch & $\$ 5.00$ & $8 / 86$ \\
\hline $\begin{array}{l}\text { 17. Foundations of } \\
\text { Political Theory }\end{array}$ & $\begin{array}{l}\text { Chair: Ronald Terchek, } \\
\text { Gov. and Pol., Univ. of } \\
\text { Maryland, College Park, } \\
\text { MD 20740; (301) } \\
454-2247 \\
\text { Newsletter Editor: } \\
\text { Ronald Terchek }\end{array}$ & $\begin{array}{l}\text { George J. Graham, Jr., } \\
\text { Dept. of Pol. Sci., Box } \\
\text { 1814-B, Vanderbilt Univ., } \\
\text { Nashville, TN 37235; } \\
\text { (615) 322-6222 } \\
\text { Program Organizer-Elect: } \\
\text { Robert Grafstein, Univ. } \\
\text { of Georgia, Pol. Sci., } \\
\text { Athens, GA 30602; } \\
\text { (404) 542-2057 }\end{array}$ & $\$ 8.00$ & $4 / 87$ \\
\hline 18. Computer Uses & $\begin{array}{l}\text { President: Stephen } \\
\text { Frantzich, Dept. of Pol. } \\
\text { Sci., U.S. Naval Acad., } \\
\text { Annapolis, MD 21402; } \\
\text { (301) 267-3170 } \\
\text { President-Elect: Michael } \\
\text { Vasu, No. Carolina } \\
\text { State Univ., Box } 8101 \text {. } \\
\text { Raleigh, ND } 27695 ; \\
(919) 737-3791 \\
\text { Newsletter Editor: } \\
\text { Michael Vasu } \\
\text { Financial Officer: J. } \\
\text { Michael Thompson, } \\
\text { Dept. of Pol. Sci., No. } \\
\text { Kentucky Univ., Highland } \\
\text { Heights, KY } 41076 ; \\
\text { (606) } 572-5326 \\
\text { Executive Council: Stuart } \\
\text { Nagel, Univ. of Illinois; } \\
\text { Ken janda, Northwest- }\end{array}$ & Michael Vasu & $\$ 5.00$ & $4 / 88$ \\
\hline
\end{tabular}




\begin{tabular}{lll} 
Name of Section $\quad$ Contact or Officers & $\begin{array}{l}\text { Annual Meeting } \\
\text { Program Organizer }\end{array} \quad$ Dues Estab. \\
\hline
\end{tabular}

ern Univ:; Charles Ben-

jamin, Bethel College;

Bob Farzanegan, Univ. of

No. Carolina, Asheville;

Bob Loevy, Colorado

Coll.; Joe Oppenheimer,

Univ. of Maryland; Helen

Purkitt, U.S. Naval Acad.

\begin{tabular}{lllll}
\hline 19. International & Chair: Paul R. Viotti, U.S. & $\$ 5.00$ & $9 / 88$ \\
Security and & Air Force Acad., Box & & \\
Arms Control & 138, U.S. Air Force & & & \\
& Acad., CO 80840; (303) & & \\
& $472-2270$ & & & \\
\hline 20. Comparative Politics & Chair: Peter Lange, Duke & Peter Lange & & \\
& Univ., Pol. Sci., 214 & & & \\
& Perkins, Durham, NC & & \\
& 27706; (919) 684-2916 & & \\
\hline
\end{tabular}

The Council approved the establishment of Sections in principle at its September 1981 meeting; Section guidelines were approved at the September 1982 meeting; the first Section was approved at the March 1983 Council meeting.

\section{GUIDELINES FOR APSA SECTIONS}

1. The Council will act on recognizing a Section upon receipt of a pledge of membership of at least 100 Association members, which includes the proposed Section name, statement of purpose, and procedures for governance (see item 6 below). The pledge of membership will be in the form of a card with name, address, signature. Cards will be supplied by the APSA.

2. All Section members must be APSA members.

3. The APSA will solicit Section memberships and collect Section dues (in conjunction with its regular membership and renewal activities), maintain Section lists, produce Section lists, produce Section rosters and mailing labels, permit Sections to sponsor panels at the annual meeting, help support the publication of newsletters, and publish Section notices in PS. In addition, the APSA will seek to assist Sections with more ambitious activities, such as conferences and publications that require external funding.

4. A basic Section dues of $\$ 3.00$ will be assessed and retained by APSA to cover the costs of the specific services listed in item 3 above. Sections may set dues at a higher level and retain the balance to finance newsletters and other activities.

5. Panels sponsored by Sections will constitute Part Two of the official Annual Meeting program, Part One being composed of panels organized by the Program Committee. For the present, there is no change in the Council policy that provides meeting rooms (on a space available basis) and courtesy listings to unaffiliated groups. APSA's Council has approved the integration of Organized Section panels in Part One of the Program for 1989.

6. Sections are required to adopt procedures that provide for an elected president or chair, an elected representative council or executive committee and an elected or appointed financial officer who is responsible for submitting an annual report to APSA's executive director. 
7. A Council Committee on Sections will recommend action on petitions to establish Sections, monitor Section activities, advise the staff on administrative arrangements for Sections, and conduct an ongoing review of Sections.

8. The Council will withdraw approval for Sections failing to maintain a membership of 100 over a period of two years. 